Jose Guido Martinez Amador.' (D)

Evelyn Verónica Soria Quiroz.2 (D)

Daiana Beatriz Ramos Medrano. ${ }^{2}$ (D)

Nelson Abel Fernandez Zambrana. $^{3}$ (D)

Luis Fernando Rojas

Terrazas. ${ }^{2,4}$ (ID)

Correspondencia a:

1 Medico Otorrinolaringólogo, Medico Aeronáutico, Instituto de audiología Cochabamba, Jefe médico Centro medico aeronáutico $N^{\circ} 2$, Docente de la materia de otorrinolaringología Universidad técnica privada cosmos UNITEPC.

Cochabamba - Bolivia

${ }^{2}$ Centro de investigación medicina UNITEPC. CIMU.

Cochabamba - Bolivia

${ }^{3}$ Docente de la materia de acústica de locales en la carrera de ingeniería de sonido UNITEPC.

Cochabamba - Bolivia

${ }^{4}$ Centro de investigación de salud publica UNITEPC. CISPU.

Cochabamba - Bolivia

Email de contacto:

jomartines1933@unitepc-mail.com

Irojas_investigacion@unitepc-mail.com

Procedencia y arbitraje:

No comisionado, sometido a arbitraje externo

Recibido para publicación: 20 de Mayo del 2019 Aceptado para publicación: 28 de Julio del 2019

Citar como:

$\mathrm{Re} \mathrm{Ci} \mathrm{Sa}$ UNI

2019;6(2):8-13

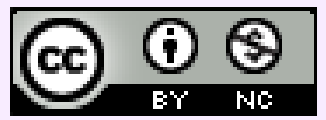

Esta obra está bajo una Licencia Creative Commons Atribución-NoComercial 4.0 Internacional.

\section{Percepción de fuentes y nivel de ruido}

\author{
Source perception and noise level
}

Percepção da fonte e nível de ruído

\section{Resumen}

Introducción: El ruido es un potencial de impacto en la salud, actualmente se considera como un contaminante "invisible" y resulta molestoso al individuo y a la población en general, el objetivo es determinar el nivel de ruido y fuentes emisoras de contaminación en la población próxima al aeropuerto de Cochabamba, Bolivia. Metodología: El estudio es descriptivo, transversal, observacional y prospectivo. Desarrollado de enero a mayo del 2019, la población de estudio contemplo 106 habitantes mayores a 15 años y el instrumento utilizado fue un sonómetro integrador tipo 2 y encuesta. Resultados: El ruido principalmente proviene del tráfico vehicular $51,9 \%$ y transporte aéreo $25,5 \%$. El $56,6 \%$ de los habitantes percibe el ruido todos los días, $31,1 \%$ los fines de semana y $12,3 \%$ de lunes a viernes. Los registros son: $90 \mathrm{~dB}$ en despegue de aviones, $86 \mathrm{~dB}$ tráfico vehicular, $105 \mathrm{~dB}$ en actividades recreativas y $65 \mathrm{~dB}$ en construcciones y locales Nocturnos. Discusión: La percepción de fuente de ruido más importante en el área del aeropuerto Jorge Wilsterman es el transporte vehicular e indicaron que es todos los días de la semana y la fuente emisora de mayor intensidad son las actividades recreativas. La mayoría de los niveles registrados en las fuentes sonoras superan los valores recomendados por la OMS e instituciones gubernamentales.

Palabras claves: Ruido, Percepción Auditiva, Contaminación por Tráfico Vehicular, Aeropuertos, Actividades Recreativas, Salud, Ambiente.

\section{Summary}

Introduction: Noise is a potential impact on health, it is currently considered as an "invisible" pollutant and it is annoying for a person and the population in general, the objective is to determine the noise level and the pollution sources in the population that lives near the Cochabamba's airport in Bolivia. Methodology: The study is descriptive, transversal, observational and prospective. Developed from January to May 2019, the study population contemplated 106 inhabitants older than 15 years and the instrument used was a type 2 integrating sound level meter and survey. Results: The noise mainly comes from vehicular traffic $51.9 \%$ and air transport $25.5 \%$. the $56.6 \%$ of inhabitants perceive noise every day, $31.1 \%$ on weekends and $12.3 \%$ from Monday to Friday. The records are: $90 \mathrm{~dB}$ in aircraft takeoff, $86 \mathrm{~dB}$ vehicular traffic, $105 \mathrm{~dB}$ in recreational activities 
and $65 \mathrm{~dB}$ in buildings and nightclubs. Discussion: The most important source of noise perception, in the Jorge Wilsterman airport area is the vehicular transport and they indicated that it is every day in the week and the greatest intensity source are the recreational activities. The Most levels recorded in the sound sources exceed the values recommended by WHO and government institutions..

Keywords: Noise, Auditory Perception, Traffic-Related Pollution, Airports, Leisure Activities, Health, Environment.

\section{Resumo}

Introdução: O ruído é um problema de potencial impacto na saúde. Atualmente é considerado um poluente "invisível" e é irritante para os indivíduos e a população em geral, O objetivo é determinar o nível de ruído e as fontes de poluição na população perto do aeroporto de Cochabamba, Bolívia. Metodologia: O estudo é descritivo, transversal, observacional e prospectivo. Desenvolvido no período de janeiro a maio de 2019.A população do estudo teve um total de 106 habitantes com idade superior a 15 anos e o instrumento utilizado foi um medidor de nível sonoro e sonda de integração tipo 2. Resultados: O ruído provém principalmente do tráfego de veículos $51,9 \%$ e do transporte aéreo de $25,5 \%$. Somente $56,6 \%$ dos habitantes percebem ruído todos os dias, $31,1 \%$ nos finais de semana e $12,3 \%$ de segunda a sexta-feira. Os registros são: $90 \mathrm{~dB}$ na decolagem de aeronaves, $86 \mathrm{~dB}$ em tráfego de veículos, 105 $\mathrm{dB}$ em atividades recreativas e $65 \mathrm{~dB}$ em prédios e boates. Discussão: A percepção da fonte mais importante de ruído na área do aeroporto Jorge Wilsterman é o transporte de veículos e indicou que ocorre todos os dias da semana sendo que a fonte de maior intensidade são as atividades recreativas. A maioria dos níveis registrados nas fontes sonoras excede os valores recomendados pela OMS e pelas instituições governamentais.

Palavras chave: Ruído, Percepção Auditiva, Poluição Relacionada com o Tráfego, Aeroportos, Atividades de Lazer, Saúde, Meio Ambiente

\section{Introducción}

La organización Mundial de la Salud y otros organismos internacionales, declaran de manera unánime que el ruido es un potencial de impacto en la salud $(1,2)$.

En el mundo el ruido es un clúster de sonidos de varias frecuencias que actualmente se considera como un contaminante "invisible" y resulta molestoso al individuo y a la población en general, el cual origina preocupación principalmente en el área urbana por el tráfico ocasionado debido a la cantidad de vehículos, el uso de bocinas, reproductores de sonido con volúmenes elevados y transporte aéreo entre otros (3).

La fuente emisora es cualquier objeto o artefacto que genera una onda sonora, ya sea de tipo estacionario, móvil o portátil (4).

En el mundo 466 millones de personas padecen pérdida de audición, principalmente niños, en este sentido se proyecta que para el 2050 aproximadamente 900 millones de personas sufrirán pérdida de audición; los factores pueden ser prevenibles en un $60 \%$ entre ellos está, la disminución de empleo de algunos fármacos ototóxicos, exposición excesiva al ruido e infecciones crónicas del oído (5)

El marco legal en Bolivia la ley del Medio ambiente 1333 de 1992, establece normas 
de protección y conservación de la salud auditiva, buscando el bienestar de la población debido a la producción y emisión de ruido (6).

En el artículo 18 del capítulo 1 de calidad ambiental indica: "El control de la calidad ambiental es de necesidad y utilidad pública e interés social. La secretaría nacional y las secretarías departamentales del Medio Ambiente promoverán y ejecutarán acciones para hacer cumplir con los objetivos del control de la calidad ambiental" (6).

Asimismo, en el capítulo III aire y atmósfera, artículo 42 menciona: "El Estado, a través de sus organismos competentes, establecerá, regulará y controlará los niveles de ruidos originados en actividades comerciales, industriales, domésticas, de transporte u otras a fin de preservar y mantener la salud el bienestar de la población" (6)

Según estimaciones internacionales el ruido en ambientes urbanos es generado por; tráfico vehicular, industrias, ferrocarriles, tráfico aéreo y actividades recreativas (1).

Las fuentes de ruido son numerosas en la actualidad, ya que son manifestaciones de energías liberadas que producen altos niveles de ruido que tienen efectos en la salud. Podemos mencionar que los problemas podrían ser: Trauma acústico en el oído interno, hipertensión arterial, enfermedades cardiovasculares, conductas agresivas, y síntomas psiquiátricos $(2,8)$

La población en particular próxima al aeropuerto Jorge Wilsterman no toma en cuenta las condiciones ambientales y realiza construcciones muchas veces con fines comerciales y se observa que está densamente urbanizando, hoy en día se tiene que reconocer la importancia de la contaminación ambiental principalmente la sonora en el diseño y la reglamentación de urbanización como factor decisivo del bienestar humano.

Por lo tanto, el estudio tiene como objetivo; Determinar el nivel de ruido y fuentes emisoras de contaminación en la población próxima al aeropuerto Jorge Wilsterman de Cochabamba, Bolivia.

\section{Metodología}

El estudio realizado es; descriptivo, transversal, observacional y prospectivo. Realizado en enero a mayo del 2019. Las mediciones se tomaron cerca de la malla de seguridad del aeropuerto Jorge Wilsterman de Cochabamba Bolivia, entre las 13:00 a 19:00 horas, exceptuando locales nocturnos que fueron evaluados entre 21:00 a 02:00 horas.

La población de estudio contemplo 106 habitantes mayores a 15 años que cumplieron con los criterios de inclusión y exclusión previamente establecidos.

El instrumento utilizado fue un sonómetro integrador tipo 2 (margen de error $\pm 1 \mathrm{~dB}$ ), debidamente calibrado. Los resultados de las mediciones se registraron en decibeles $(\mathrm{dB})$, además se utilizó un cuestionario para establecer las fuentes de ruido que reconocen las personas y que días de la semana es más acentuado este problema.

Los datos fueron procesados en el programa IBM-SPSS Statistics versión 25 y se procedió con el análisis descriptivo de las variables.

La recolección de la información fue autorizada por el representante de la organización territorial de base y los propios encuestados. 


\section{Resultados}

En el cuadro 1 se observa que la población próxima al aeropuerto, refiere que el ruido principalmente proviene del tráfico vehicular $51,9 \%$, seguido del transporte aéreo $25,5 \%$ y el restante $22,6 \%$ por otras (bocinas y parlantes de vehículos).

Cuadro 1. Percepción del ruido de habitantes que viven cerca de la malla de seguridad del aeropuerto Jorge Wilsterman

\begin{tabular}{|l|c|c|}
\hline Fuentes de ruido & Frecuencia & Porcentaje \\
\hline Tráfico vehicular & 55 & 51,9 \\
\hline Transporte Aéreo & 27 & 25,5 \\
\hline Otros & 24 & 22.6 \\
\hline Total & $\mathbf{1 0 6}$ & $\mathbf{1 0 0 , 0}$ \\
\hline
\end{tabular}

Fuente: Propia de los autores

En el cuadro 2 se observa, que días de la semana se percibe más ruido: El 56,6\% de los habitantes percibe el ruido todos los días, seguido de $31,1 \%$ los fines de semana y el restante $12,3 \%$ de lunes a viernes.

Cuadro 2: Que días perciben ruido durante la semana.

\begin{tabular}{|l|c|c|}
\hline Días de la semana & Frecuencia & Porcentaje \\
\hline Lunes a viernes & 13 & 12,3 \\
\hline Fines de semana & 33 & 31,1 \\
\hline Todos & 60 & 56,6 \\
\hline Total & $\mathbf{1 0 6}$ & $\mathbf{1 0 0 , 0}$ \\
\hline
\end{tabular}

Fuente: Propia de los autores

Los registros obtenidos de distintas fuentes sonoras a las que está expuesta la población son: $90 \mathrm{~dB}$ en despegue de aviones, $86 \mathrm{~dB}$ tráfico vehicular, $105 \mathrm{~dB}$ en actividades recreativas (fiestas) y $65 \mathrm{~dB}$ en construcciones y locales Nocturnos. Ver gráfico 1.

Grafico 1: Nivel en decibeles de fuentes sonoras

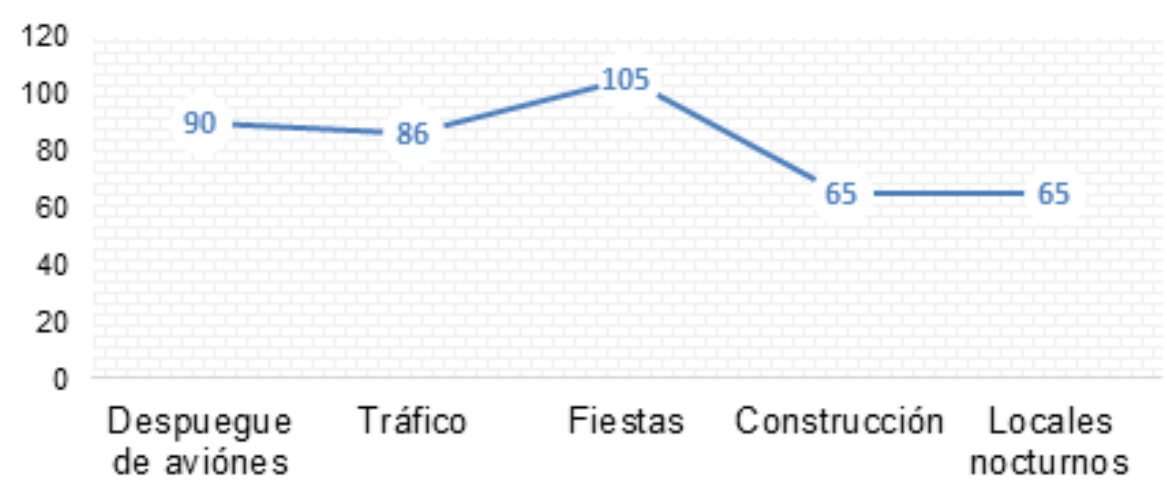

Fuente: Propia de los autores

\section{Discusión}

La población que vive próxima al aeropuerto refiere que el ruido principalmente proviene del tráfico vehicular $51,9 \%$, que se relaciona con otros estudios como el realizado en Puerto Montt Chile en donde indica $47.6 \%$, en segundo lugar, está el transporte aéreo $25,5 \%$ no hay estudios para comparación en este punto (9). 
El $56.6 \%$ de los habitantes refieren qué perciben ruido todos los días, es elevado en relación con otro estudio realizado en Chile, donde indica $43.7 \%$. Los fines de semanas fue $31.1 \%$ el cual es similar con el trabajo citado anteriormente $32.6 \%$. De lunes a viernes es $12.3 \%$ por debajo con el trabajo en comparación $23.7 \%$. Se debe tomar en cuenta en este punto la percepción de ruido es subjetiva y a esto se deba la diferencia de los resultados de nuestro trabajo en relación con otros (10).

El parámetro establecido por la Organización Mundial de la Salud (OMS) es ruido no mayor a $55 \mathrm{~dB}$ y también sugieren valores adicionales para ambientes específicos. La norma boliviana establece 68 decibeles en el día y de 65 durante la noche como límite máximo permisible y en el municipio de Cercado Cochabamba se indica durante el día la intensidad de ruido generado por un local de diversión y transmitido hacia el exterior no deberá superar los 68 decibeles, en la noche no deberá ser mayor a los 65 decibeles, en cumplimiento al Decreto Reglamentario de la Ley $1333(6,11)$.

En relación con los registros obtenidos muestran que al despegar aviones la intensidad es de $90 \mathrm{~dB}$, y este valor se relaciona con el trabajo realizado en el aeropuerto de Palma de Mallorca España donde indican de 90 a $100 \mathrm{~dB}$, la OMS no especifica valores para aeropuertos (12)

En relación a el tráfico vehicular la medición mostró $86 \mathrm{~dB}$, es elevado en relación con valores guía de la OMS $70 \mathrm{~dB}$.

Las actividades recreativas (fiestas), son los ambientes donde se presentan datos elevados $105 \mathrm{~dB}$, que está $5 \mathrm{~dB}$ encima de los valores guía de la OMS $100 \mathrm{~dB}$.

Los valores tomados en construcciones y locales nocturnos es de 65 dB que están 5 $\mathrm{dB}$ debajo de los valores indicados por la OMS $70 \mathrm{~dB}$.

En conclusión, la percepción fuente de ruido de mayor importancia en el área del aeropuerto Jorge Wilsterman, es el transporte vehicular, también indicaron que es todos los días de la semana y la fuente emisora de mayor intensidad son las actividades recreativas (fiestas)

La mayoría de los niveles registrados e las fuentes sonoras superan los valores recomendados por la OMS e instituciones gubernamentales. Se recomienda al municipio de Cochabamba tomar medidas ante la contaminación acústica, y colocar señales de seguridad para advertir a la población sobre el peligro.

\section{Conflictos de Intereses}

Los autores declaramos no tener conflictos de interés para el presente estudio.

\section{Referencias Bibliográficas.}

1. OMS. 1100 millones de personas corren el riesgo de sufrir pérdida de audición [Internet]. [citado 14 de mayo de 2019]. Disponible en: https://www.who.int/mediacentre/news/releases/2015/ear-care/es/

2. Sociedad Española de Acústica. Día Internacional de Concienciación sobre el Ruido. [Internet]. [citado 14 de mayo de 2019]. Disponible en: http://www.sea-acustica. es/index.php?id=44

3. Peligros del ruido y sus efectos en nuestra salud [Internet]. [citado 14 de mayo de 2019]. Disponible en: https://www.elsevier.com/es-es/connect/actualidad-sanitaria/ 
efectos-negativos-del-ruido-y-su-repercusion-en-nuestra-salud

4. Santos De La Cruz E. Contaminación sonora por ruido vehicular en la avenida Javier Pardo. Ind. data. 2007; 10(1):11-15. doi: https://doi.org/10.15381/idata. v10i1.6201

5. OMS. Sordera y pérdida de la audición [Internet]. [citado 14 de mayo de 2019]. Disponible en: https://www.who.int/es/news-room/fact-sheets/detail/deafness-and-hearing-loss

6. Leyes y Normas. Ministerio de Medio Ambiente y Agua [Internet]. [citado 14 de mayo de 2019]. Disponible en: https://www.mmaya.gob.bo/marco-legal/leyes-y-normas/

7. Esteban Alonso A de. Contaminación acústica y salud. Obs. Medioambient. 2003; 6(1): 73-95

8. Observatorio de salud y medio ambiente de Andalucia. Ruido y salud. [Internet]. [citado 14 de abril de 2019]. Disponible en: https://www.diba.cat/c/document_library/get_file?uuid=72b1d2fd-c5e5-4751-b071-8822dfdfdded\&groupld=7294824

9. Vega VHL, Silva EAS. Estudio subjetivo de la percepción del ruido ambiental en la ciudad de Puerto Montt. 2008;2-8.

10. Vega VHL. Evaluación del ruido ambiental en la ciudad de Puerto Montt [tesis de grado]. Valdivia Chile: Universidad austral de Chile; 2008

11. OMS. Guías para el ruido urbano [Internet]. [citado 19 de mayo de 2019]. Disponible en: http://www.juristas-ruidos.org/Documentacion/guia_oms_ruido_1.pdf

12. Pons JMS, Reynés MRM, Pérez MR, Peñas JEM. El problema del ruido en los entornos aeroportuarios. El caso del aeropuerto de Palma de Mallorca. BAGE [Internet]. 2004 [citado 19 de mayo de 2019];38. Disponible en: https://www.age-geografia.es/ojs/index.php/bage/article/view/490 